32 | 2001

L'épopée orale turque d'Asie centrale. Contes épiques nanaïs

\title{
I. Bref aperçu de l'épopée orale turque en Asie centrale
}

I. A Brief Survey of the turkic Oral Epic of Central Asia

Karl Reichl

\section{(2) OpenEdition}

\section{Journals}

Édition électronique

URL : https://journals.openedition.org/emscat/1263

DOI : 10.4000/emscat.1263

ISSN : 2101-0013

Éditeur

Centre d'Etudes Mongoles \& Sibériennes / École Pratique des Hautes Études

Édition imprimée

Date de publication : 1 décembre 2001

Pagination : 9-37

ISBN : 2-9518888-0-5

ISSN : 0766-5075

Référence électronique

Karl Reichl, «I. Bref aperçu de l'épopée orale turque en Asie centrale », Études mongoles et sibériennes, centrasiatiques et tibétaines [En ligne], 32 | 2001, mis en ligne le 17 mars 2009, consulté le 13 juillet 2021. URL : http://journals.openedition.org/emscat/1263 ; DOI : https://doi.org/10.4000/emscat.1263

Ce document a été généré automatiquement le 13 juillet 2021.

(c) Tous droits réservés 


\title{
I. Bref aperçu de l'épopée orale turque en Asie centrale
}

\author{
I. A Brief Survey of the turkic Oral Epic of Central Asia
}

Karl Reichl

\section{NOTE DE L'AUTEUR}

La présente étude se fonde sur des conférences données à l'École pratique des hautes études, section des Sciences religieuses, au printemps de 1995. Je remercie Madame Roberte Hamayon de m'avoir donné l'occasion de présenter ces conférences en qualité de directeur d'études invité. Mes remerciements vont aussi aux auditeurs de mes conférences, étudiants ou collègues, dont les interventions lors des discussions ont contribué à l'ambiance stimulante de l'E.P.H.E. Enfin, je remercie Mesdames Marie-Lise Beffa et Laurence Delaby des soins apportés à revoir mon style. Des fautes qui pourraient rester, je porte seul la responsabilité.

1 Des steppes aux océans est le titre d'une étude de synthèse sur l'indo-européen et les «Indo-Européens» due à André Martinet, professeur émérite à l'université René Descartes et directeur d'études à l'École pratique des hautes études ${ }^{1}$. Dans cet ouvrage majeur, Martinet donne une ébauche de la structure et du développement des langues indo-européennes, ainsi que de leur origine et de leur expansion. Nées dans les steppes de l'Eurasie, les langues indo-européennes s'étendaient déjà à l'époque préhistorique jusqu'à l'océan Indien, la Méditerranée et l'Atlantique. De nos jours, elles occupent un vaste territoire distribué sur tous les continents et bordé par tous les océans. Le même intitulé, Des steppes aux océans, pourrait aussi servir de titre à un livre sur les langues turques et le monde des peuples turcs ${ }^{2}$. Le berceau de cette famille de langues se situe probablement aussi dans les steppes : steppes de la Sibérie méridionale, aux pentes de l'Altaï, d'où peuples et langues turcs se répandirent jusqu'aux océans, océan glacial Arctique (mer des Laptev) au nord-est et Méditerranée au sud-ouest. Avec une étendue de plus de huit mille kilomètres à vol d'oiseau depuis la Iakoutie au nord-est de la 
Sibérie jusqu'aux Turcs de la péninsule balkanique, ce "monde turc" offre une abondante variété de cultures, de religions et de structures sociales. Presque toutes les grandes religions du monde y sont (ou y ont été) représentées: bouddhisme, manichéisme, judaïsme, islam (sunnite et chiite), christianisme (orthodoxe et nestorien) et chamanisme. Il y a dans ce "monde turc" de vieilles civilisations raffinées comme celle des Ouïghours du bassin du Tarim (Xinjiang, VIII ${ }^{\mathrm{e}}$-XIV ${ }^{\mathrm{e}}$ siècles). De cette civilisation se sont conservées les traces d'une riche littérature, à caractère religieux principalement (bouddhisme, manichéisme), et d'un art florissant - dont témoignent les peintures murales de Dunhuang, Bäzäklik et autres sites du nord-ouest de la Chine. Mais dans ce monde on rencontre aussi des petits peuples comme les Turcs de l'Altaï (Altaïens, Khakasses, Chors et autres), qui ont préservé jusqu'au $\mathrm{xx}^{\mathrm{e}}$ siècle l'organisation sociale d'une culture de chasseurs, caractérisée dans le domaine spirituel et intellectuel par la prédominance du chamanisme et l'absence totale d'une littérature écrite ${ }^{3}$.

$2 \mathrm{Au}$ vu de cette diversité, il est problématique de parler de "l'épopée turque » ou de «l'épopée orale turque » tout court, et même - malgré la restriction géographique de «l'épopée orale turque d'Asie centrale ». Il y a, à vrai dire, une multitude de traditions épiques turques, qui mériteraient toutes une étude spéciale. Mais comme les langues turques se ressemblent de manière frappante - à la seule exception du tchouvache (parlé par les Tchouvaches des bords de la moyenne Volga) -, il existe aussi dans les traditions épiques des peuples turcs des constantes qui, en dépit de la diversité apparente, laissent deviner leurs racines communes. Au surplus, on rencontre, entre certaines traditions, des ressemblances étroites, qui s'expliquent par leur histoire partagée. Ceci est vrai des traditions épiques des Kirghizes, Kazakhs, Karakalpaks et Ouzbeks (nomades). L'âge héroïque de l'épopée kazakhe et karakalpake, par exemple, est le temps de la Horde des Noghaïs, c'est-à-dire l'époque où ces peuples, ou plutôt les tribus qui les composaient, faisaient encore partie d'une confédération tribale commune, qui ne fut dissoute qu'à la fin du $\mathrm{xvI}^{\mathrm{e}}$ siècle. Avant de discuter les caractéristiques les plus importantes communes aux traditions épiques turques d'Asie centrale, il est peut-être utile de présenter ces traditions ${ }^{4}$.

3 Sans s'attacher aux détails, on distingue en gros une demi-douzaine de zones principales sur la carte de l'épopée turque orale. Il y a d'abord, au nord-est de la Sibérie, l'épopée des Iakoutes (appelée oloñxo), caractérisée par sa nature archaïque et son rattachement étroit au chamanisme. Plus au sud, les traditions épiques des peuples turcs de l'Altaï sont, à cause de leur lien avec le chamanisme, assez semblables à celle des Iakoutes. Un troisième groupe est formé par les épopées turques d'Asie centrale au sens strict, c'est-à-dire celles des Kirghizes, des Kazakhs, des Karakalpaks, des Ouzbeks et des Ouighours. Ce groupe n'est pas tout à fait homogène; il s'y trouve un grand nombre de genres épiques : épopée héroïque et épopée lyrique, épopée en vers et récit où prose et vers se mêlent (prosimetrum en latin médiéval), brève chanson héroïque et épopée de grande dimension, comparable en longueur aux épopées homériques ou même indiennes. Ces traditions, que je nommerai «traditions centrales", sont contiguës à une quatrième zone, domaine de la poésie épique des Turcs du sud-ouest : Turkmènes, Azerbaïdjanais et Turcs de Turquie. Là, la forme "prosimétrique » et l'épopée lyrique prévalent. À ces quatre zones, il faut ajouter la tradition épique orale des Bachkirs, qui habitent le sud de l'Oural. L'épopée bachkire se rapproche, par sa forme et son contenu, tant de l'épopée sibérienne que de celle des traditions centrales. 
Elle présente aussi des ressemblances avec les récits épiques des Tatars. Ces derniers, comme les Bachkirs, relèvent linguistiquement du groupe kiptchak et sont donc apparentés aux Kazakhs et aux Karakalpaks, avec lesquels ils partagent un certain nombre de légendes et de traditions historiques. À ce sous-groupe appartiennent aussi les Noghaïs, un des peuples turcophones du Caucase du Nord (Koumyks, Karatchaïs et Balkars). Il est intéressant de noter qu'une partie de la poésie épique des ces peuples en particulier les chansons narratives et les récits héroïques des Karatchaïs et des Balkars - se rattache aux légendes des Nartes, répandues surtout chez les Ossètes (iranophones), mais aussi chez d'autres peuples caucasiens.

Pour mieux mettre en évidence les caractéristiques de l'épopée orale turque, je citerai quelques exemples pris dans les «traditions centrales ». C'est dans cette zone que j'ai fait mes recherches, chez les Ouzbeks, les Karakalpaks, les Kazakhs et les Kirghizes, et ma discussion de l'épopée turque aura donc principalement trait à ces traditions. Je prendrai néanmoins aussi en considération la région sibérienne de l'Altaï, notamment quand j'aborderai la question des strates chamaniques dans l'épopée orale des peuples turcs. Voici en guise d'introduction un bref extrait d'une épopée karakalpake :

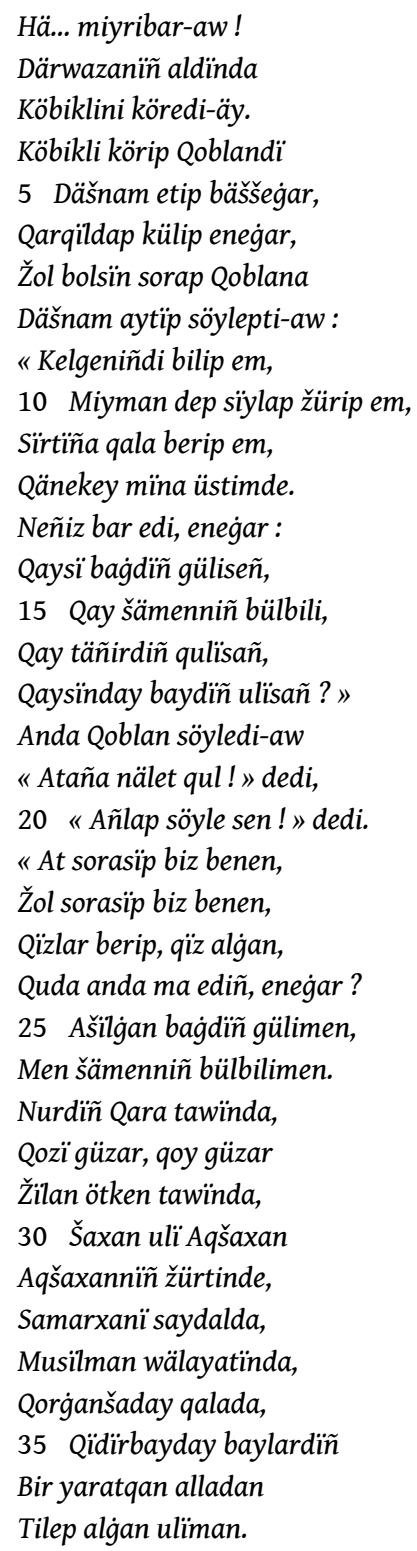


Din qilišim qalmaqqa,

Qalmaqti dinge salmaqqa

40 At oynatïp kelgenmen.

Meniñ atïm sorasañ,

Senler kibi däwlerdiñ

Sallagïin, Qoblan bolurman. $»^{5}$

Ô... Dieu !

Devant le portail [de la ville d'Azïw]

Il [Qoblan] vit [le géant] Köbikli.

Quand Köbikli vit Qoblan,

5 Le coquin dit des injures,

Le fripon s'esclaffa

Et demanda à Qoblan où il allait,

L'insulta et dit :

« Je te vois venir,

10 Je t'offre mes services comme à un hôte,

Je te donne ma ville,

La voilà, à ton service.

Qui es-tu, fripon?

De quel jardin es-tu la fleur?

15 De quel pré es-tu le rossignol?

De quel dieu es-tu l'esclave?

De quel bay [homme riche] es-tu le fils?»

Alors Qoblan répondit :

«Sacré esclave! » dit-il,

20 « Parle avec raison! » dit-il.

«Si tu me demandes mon nom,

Si tu me demandes ma route,

Si tu me donnes tes filles et que je les prends,

Seras-tu alors mon beau-père, coquin?

25 Je suis en effet la fleur florissante d'un jardin, Je suis le rossignol d'un pré.

Au Mont Noir de Nour,

Au Gué-des-agneaux et au Gué-des-brebis,

$\mathrm{Au}$ Mont-où-rampe-le-serpent,

30 Dans le pays d'Aqšaxan,

Du fils de Šaxan,

Dans les environs de Samarkand,

Dans le pays musulman,

Dans la ville de Qorganša,

35 Je suis le fils, désiré

Du seul Dieu,

Du bay Qïdïrbay.

Mon sabre de religion est pour les Kalmouks,

Pour convertir les Kalmouks

40 Je suis venu sur mon cheval, le faisant caracoler.

Si tu demandes mon nom :

Je suis le bourreau de monstres

Comme vous, je suis Qoblan. »

5 Cet extrait provient de l'épopée Qoblan et fut chanté par le barde karakalpak Žumabayžïraw Bazarov. Le contexte est le suivant: Qoblan, fils du vieux Qïdïrbay, s'était attiré l'inimitié d'Aqšaxan. Pour détruire Qoblan, Aqšaxan l'envoie à Azïw, ville où règne le géant Köbikli (appelé däw, du persan dîw). Quand Köbikli aperçoit Qoblan, il éclate de rire et l'accoste sur un ton ironique. Qoblan révèle son identité et ne laisse aucun doute 
sur ses intentions hostiles. Par la suite, Qoblan vaincra Köbikli, mais ce ne sera pas une victoire facile ${ }^{6}$.

6 Žumabay-žïraw est né en 1927 à Šomanay en Karakalpakie. C'est un barde professionnel qui a acquis son art au cours d'un apprentissage auprès d'un autre barde karakalpak, Esemurat-žïraw. Pendant cette période, qui dura trois ans, Žumabay apprit les épopées Qoblan, Edige et Šaryar. Ce sont de longues épopées, dont la récitation s'étend sur deux ou trois nuits (par épopée). À la fin de l'apprentissage, le maitre barde donna sa bénédiction (appelée pätiya en karakalpak, de l'arabe fatiha) à son élève. Cette façon d'apprendre le répertoire est typique des traditions centrales. On peut ainsi retracer des "généalogies » de bardes, c'est-à-dire les répartir en «écoles de bardes » d'après les lignées de transmission orale. En Ouzbékistan, par exemple, on compte plusieurs de ces « écoles de bardes » (baxši maktablari), dont les deux plus importantes sont l'« école de Bulungur » et l'« école de Kattaqorḡān », la première étant renommée pour son style héroïque, et la seconde pour son style lyrique. Selon les spécialistes du folklore de l'Ouzbékistan, l'arbre généalogique de l'« école de Kattaqorgān » remonte au XVII e siècle à un barde nommé Yādgār-baxši, duquel dérivent plus de cinquante bardes ouzbeks ${ }^{7}$.

7 La généalogie des bardes (Žiraw) karakalpaks ne remonte, elle, qu'au début du XIX $x^{e}$ siècle, si l'on exclut un barde légendaire du xIV siècle, Soppaslï Sïpïra-žïraw. Žumabay-žïraw continue l'art de son maître Esemurat-žïraw (1893-1979) qui lui-même avait été l'élève de son père, Nurabulla-žïraw (1862-1922). Nurabulla avait appris l'art de chanter l'épopée de Turïmbet-žïraw (à Šïmbay) et, par la suite, il enrichit son répertoire en se faisant šägirt (élève) de plusieurs bardes, en particulier de Qazaqbay à Boukhara $^{8}$. Ces relations entre bardes, parfois assez complexes, expliquent les ressemblances ainsi que les divergences entre les différentes versions d'une même épopée. Malheureusement, la documentation est en général si lacunaire qu'une affiliation des textes notés par "école » et par voie de transmission n'est possible qu'exceptionnellement.

8 Le répertoire des bardes ne comporte pas seulement des épopées ; celui de Žumabayžïraw comporte aussi des poèmes plus courts, comme un poème-louange à sa ville natale Šomanay, des poèmes-conseils (nasihat) et un poème historique (tolgaw) sur un chef noghaï, Ormanbet-biy. Ces poèmes - appelés terme en karakalpak (terma en ouzbek), littéralement « collection » - ont plusieurs fonctions. Ils rendent plus varié le répertoire du barde et lui donnent la possibilité de chanter aussi lors d'occasions où le temps manque pour narrer une épopée en entier. Ces poèmes peuvent être traditionnels (tel le tolgaw sur Ormanbet-biy) ou composés par le barde lui-même (tel le poème-louange à Šomanay). Plus important est le fait que ces terme servent d'introduction à la récitation de l'épopée. Un type de terme rencontré chez les bardes ouzbeks consiste justement en une énumération du répertoire du barde. Il demande à ses auditeurs ce qu'ils veulent entendre ("Nima aytayin", "Qu'est-ce que je dois dire?», est le titre d'un terme de ce type). Ce prélude à l'épopée est aussi nécessaire pour le barde : il essaie son instrument et sa voix et - plus significatif - il se met dans l'état d'âme exalté dont il a besoin pour la récitation.

9 Žumabay Bazarov est un žiraw, terme dérivé de žir (ancien turc yïr, ïr), «chant », et signifiant littéralement "chanteur ». Ce terme (ou un dérivé analogue) se trouve aussi chez les Kazakhs et les Kirghizes9. La désignation du barde la plus répandue en Asie centrale est baxši ; ce mot est employé en ouzbek, en ouïghour, en turkmène et - pour un type de barde différent du žïraw - également en karakalpak (baqsii). L'étymologie de 
baxši est problématique; le terme se trouve déjà en turc ancien (aussi en mongol) et semble emprunté au chinois ou peut-être au sanskrit ${ }^{10}$. Comme on le verra au chapitre III, il désigne aussi le chamane ou plutôt le guérisseur chez les Kazakhs et les Kirghizes. Quelquefois on nomme le barde à l'aide d'une dérivation du terme signifiant l'épopée. Ainsi, en iakoute, oloñxo signifie "épopée » et donc oloñxohut "chanteur d'épopée » ${ }^{11}$. En kirghize, Manas est le nom du cycle épique le plus célèbre des Kirghizes et manasči a par conséquent le sens de " chanteur de Manas » et plus généralement de «barde »"

Žumabay-žïraw s'accompagne au qobïz, sorte de viole à deux cordes, semblable à la gusle serbo-croate ou au morin-xuur mongol ${ }^{13}$. En général, l'épopée orale turque est récitée par des bardes professionnels qui s'accompagnent eux-mêmes; exception notable, les bardes kirghizes et iakoutes récitent l'épopée sans accompagnement. L'instrument favori des bardes est le luth, c'est-à-dire un instrument à cordes pincées : dombira chez les bardes ouzbeks ${ }^{14}$, dutar chez les baxši turkmènes et les baqsï karakalpaks ${ }^{15}$, dombra (d'un type différent de l'instrument homonyme des Ouzbeks) chez les bardes kazakhs ${ }^{16}$ ou topšuur des qayči («chanteurs ») khakasses ${ }^{17}$. Dans la tradition khorezmienne (en Ouzbékistan), les bardes sont souvent accompagnés par un petit ensemble, qui se compose de joueurs de gidždžak (une sorte de viole), de tambourin, de flûte et même d'accordéon ${ }^{18}$.

11 La manière de réciter est très variable. D'abord, il faut dire que l'épopée est plutôt chantée que déclamée. Ce chant peut aller de la mélodie monotone ne dépassant pas la quinte dans son étendue et se répétant pour chaque vers, jusqu'à la mélodie sinueuse composée de plusieurs motifs, se déployant sur toute une strophe et pouvant même excéder l'octave ${ }^{19}$. La structure musicale est liée à la structure métrique. Dans la poésie populaire turque, la versification est syllabique ${ }^{20}$. Ce n'est que très rarement que des bardes qui ont subi l'influence de la littérature classique introduisent des vers métriques ('arūd) dans leurs épopées (comme par exemple le baxši ouzbek Ergaš Džuman-bulbul-oġli [1868-1937]). Le vers épique par excellence est, comme dans notre extrait, un vers de sept ou huit syllabes (" octosyllabe»). Ces vers se groupent selon leurs rimes (ou assonances) en ensembles irréguliers, souvent interrompus par des vers blancs : vers 5-6 (etip) bäššejar : (külip) eneġar, vers 9-11 bilip em : žürip em : berip em, vers 31 sq. zü̈rtinde : saydalda : wälayatïnda : qalada. Ces rimes ou assonances sont souvent des rimes grammaticales, c'est-à-dire que les syllabes rimantes sont des suffixes ou des formes grammaticales, tel l'affixe du cas locatif-de/-da (žürtin-de, etc.) ou le gérondif en -ip suivi de la copule (bil-ip em, etc.). Ces vers montrent aussi une tendance marquée à construire des syntagmes de manière parallèle :

Qaysï baġdïñ güliseñ,

15 Qay šämenniñ bülbili

Qay täñirdiñ qulïsañ,

Qaysïnday baydiñ ulisañ?

[...]

21 At sorasïp biz benen,

Žol sorasïp biz benen...

De quel jardin es-tu la fleur?

15 De quel pré es-tu le rossignol?

De quel dieu es-tu l'esclave?

De quel bay es-tu le fils?

[...]

21 Si tu me demandes mon nom,

Si tu me demandes ma route... 
12

$$
\begin{aligned}
& 23 \text { Qizzlar berip, qüz algan } \\
& {[\ldots . .]} \\
& 28 \text { Qozï güzar, qoy güzar } \\
& 23 \text { Si tu me donnes tes filles et que je les prends } \\
& {[\ldots . .]} \\
& 28 \text { Au Gué-des-agneaux et au Gué-des-brebis }
\end{aligned}
$$

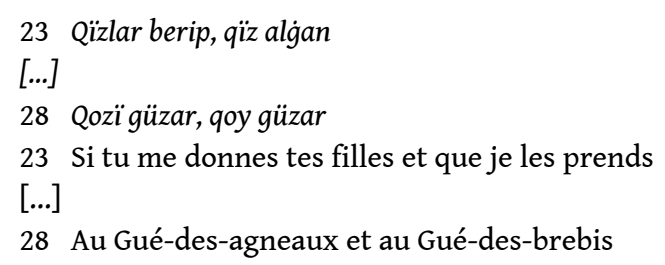

Dans l'extrait cité ces ensembles rimés (assonancés) ne sont pas très étendus; mais certains bardes et certaines traditions (surtout la kirghize) cultivent les longs passages monorimes, ce qui donne une structure métrique comparable à celle de la laisse de la chanson de geste française. Quelques vers montrent par ailleurs une autre propriété de la poésie épique, à savoir l'allitération. Les vers 14 sqq. sont liés par allitération verticale («anaphore»: qaysï, qay, qay, qaysïnday), d'autres vers présentent une allitération horizontale (comme dans la poésie en vieil anglais, allemand ou norrois) :

Dans plusieurs traditions turques (comme la kirghize et l'altaïenne), l'allitération est assez fréquente et bien développée.

14 À côté des vers "octosyllabes", il y a aussi des vers de onze ou douze syllabes («alexandrins »), souvent groupés en strophes de quatre vers. Un extrait d'une autre épopée karakalpake, venant d'un baqsï plutôt que d'un žiraw, peut illustrer ce type de vers :

1 Ešit Ğärip axu menen zarïmdï,

Yarïm kelgeniñdi bila bilmedim.

Tärk äyleyin namis bilen arïmdï,

Yarïm kelgeniñdi bila bilmedim.

5 Sen häm kelerme dep külmedim oynap,

Häsiret qazanïnda pišpedim qaynap,

Qanžar urma tände žanïmdï qüynap,

Yarïm kelgeniñdi bila bilmedim.

Döhmet äylep nahaq töxpe qanïmdï,

10 Sen men dep tärk ettim dünya malimdi.

Sen yandïrma burun žanġan žanïmdï,

Yarïm kelgeniñdi bila bilmedim.

Toy ötken soñ keldim köškim astïna,

Giyne äylep žan almaqtĩn qastïna,

15 Sänem özin taslar Gärip üstine,

Köški üstinde taqat eta bilmedim.

1 Écoute, Ġärip, mes soupirs et mes sanglots !

Je ne pouvais pas savoir que tu étais revenu, mon aimé.

Je vais abandonner mon honneur et ma vergogne,

Je ne pouvais pas savoir que tu étais revenu, mon aimé.

5 Ne sachant si tu reviendrais, je ne riais pas dans mes jeux;

Je bouillais dans le chaudron de la langueur, sans tout à fait devenir rôtie. [!]

Ne me donne pas un coup de poignard et ne prends pas ma vie!

Je ne pouvais pas savoir que tu étais revenu, mon aimé.

Ne m'accuse pas, moi qui suis innocente, et ne fais pas couler mon sang!

10 Pour me donner à toi, j'ai renoncé à toutes mes possessions.

N'allume pas mon cœur en flammes depuis longtemps !

Je ne pouvais pas savoir que tu étais revenu, mon aimé.

Après les noces, je suis venue en bas de mon palais.

Avant que tu m'accuses et prennes ma vie,

15 Sänem va se jeter sur Gärip.

Je ne peux plus patienter en haut du palais.

Études mongoles et sibériennes, centrasiatiques et tibétaines, 32 | 2001 
- c'est-à-dire d'un roman sentimental, qui narre les aventures infortunées d'un couple d'amoureux -, intitulée Gärip et Sänem ou G̈ärip-ašiq. Sänem est une jeune fille dont la main est promise dès l'enfance à Ǵärip. Après la mort du père de Ġärip, le père de Sänem s'oppose à ce mariage, puisque la famille de Ġärip est tombée dans la pauvreté. Bouleversé, Ġärip devient troubadour itinérant, un ašiq, littéralement "aimant ", comme les Turcs et les Azerbaïdjanais appellent jusqu'à nos jours les bardes et chanteurs de poésie orale. Au cours d'une intrigue complexe et fertile en aventures, Sänem, qui suppose que Gärip est mort, est forcée d'épouser un autre prétendant. Gärip, déguisé en ménestrel, arrive aux noces de Sänem juste à temps et est reconnu par sa bien-aimée. Les strophes citées se placent après la scène de la reconnaissance et précèdent le « happy end » de l'œuvre.

16 Cette épopée lyrique est l'un des récits les plus répandus du monde turc. Chez les Karakalpaks, neuf versions ont été notées, comportant entre 50 et 650 pages manuscrites $^{21}$. Il existe en outre des versions turques, turkmènes, azerbaïdjanaises, tatares (de Crimée), ouzbèkes et ouïghoures. En 1837, l'écrivain russe Mikhaïl Lermontov traduisit un récit azerbaïdjanais en russe ${ }^{22}$. Le motif central de l'histoire de Gärip et Sänem est le retour du héros, déguisé en ménestrel ou en mendiant, à point nommé, juste avant le mariage (ou le remariage) de sa bien-aimée (ou de sa femme). Ce motif est largement diffusé dans la littérature mondiale; il suffit de se rappeler le retour d'Ulysse dans L'Odyssée, scène qui trouve son équivalent exact dans l'épopée turque d'Alpamǐš ${ }^{23}$.

17 Cet extrait fut chanté en 1983 par le baqsï karakalpak Genžebay Tilewmuratov. Genžebay-baqsï est né en 1929. Son maître principal (ou ustaz, du persan ustād) fut son père, Tilewmurat-baqsï (mort en 1950). L'ustaz de son père était un barde du nom de Arzï-baqsï, chez qui Tilewmurat fit un apprentissage de huit ans, au cours desquels il apprit un répertoire de six épopées : Yusup (Yousouf) et Axmet, Sayatxan et Hamra, G̈̈ribašiq, Hürliqa et Hamra, Bäzirgen et Qïrman-däli. Genžebay avait appris à jouer du dutar dès l'âge de sept ou huit ans; à douze ans, il savait déjà par cœur les épopées Yusup et Axmet, Sayatxan et Hamra, ainsi que Qïrmandäli. De 1956 à 1960, il enseigna dans une école, plus tard il travailla pour le théâtre, la radio et la télévision en Karakalpakie ${ }^{24}$.

Quant à la structure métrique du texte, on peut remarquer que les vers comportent onze syllabes et sont organisés en quatrains. Le schéma des rimes est $a-a-a-b$; à l'exception de la dernière strophe, le dernier vers de chaque strophe est un refrain, qui est introduit au deuxième vers de la première strophe (qui rime donc $a-b-a-b)$. Cette structure s'appelle murabba', littéralement "carré », « quatrain ». Le poème est chanté sur une mélodie qui porte le nom d'« Arïwxan" (Arïwxan namasi). Selon ses dires, Genžebay-baqsï maîtrise à peu près quatre-vingts mélodies (nama). Comme on le voit, dans cette tradition l'aspect musical joue un rôle important, sinon le rôle principal. Les poèmes sont souvent chantés et transmis indépendamment des épopées, de sorte qu'aujourd'hui ils relèvent de la tradition des chansons plutôt que de la tradition épique ${ }^{25}$.

19 La manière de réciter ou de chanter les extraits d'épopées lyriques est liée à la façon dont celles-ci sont transmises. Chez les Karakalpaks, le répertoire du žiraw passe oralement d'un barde à l'autre, alors que celui des baqsï intègre aussi l'influence de versions écrites, imprimées ou manuscrites. À la fin du XIX ${ }^{e}$ siècle, nombre de récits épiques furent imprimés à Kazan afin de propager la littérature écrite parmi les

Études mongoles et sibériennes, centrasiatiques et tibétaines, 32 | 2001 
peuples turcs d'Asie centrale. Il existe aussi une tradition manuscrite, à laquelle ont surtout contribué les qissa-xān, récitants qui chantent les épopées à partir d'un texte écrit. La première édition d'extraits de Yousouf et Axmet, épopée du répertoire de Genžebay-baqsï, date de 1867 et se fonde sur un manuscrit rapporté du Khorezm par Hermann Vámbéry et qui appartenait apparemment à un qissa-xān ${ }^{26}$.

Le Khorezm est en fait un carrefour important pour les traditions épiques de l'Asie centrale. Ici se rencontrent traditions turkmènes, ouzbèkes, karakalpakes et kazakhes. Certaines « écoles » de baqsï karakalpaks ont pour maître fondateur un barde turkmène du XIX ${ }^{e}$ siècle, Süyew-baxši, qui comptait aussi un grand nombre d'élèves ouzbeks ${ }^{27}$. Il est significatif que parmi les terme des baqsï karakalpaks se trouvent aussi des poèmes du poète turkmène Makhtumkuli ( $\mathrm{du}$ XVIII ${ }^{\mathrm{e}}$ siècle). Le poème cité plus haut présente des turkménismes dans sa forme linguistique : dans la première strophe, par exemple, on a le turkmène ešit « écoute » au lieu du karakalpak esit; yar "aimé(e) » au lieu de žar; bilen «avec» au lieu de menen ${ }^{28}$. Ce mélange de transmissions orale et écrite est également révélé par une certaine stabilité du texte. La première strophe de l'extrait cité est presque identique à l'une des versions turkmènes publiées ${ }^{29}$ :

Garï xan, ešitgil meniñ zarïmï,

Seniñ geldigiñi bile bilmedim,

Terk eyledim namïs bile arïmï,

Seniñ geldigiñi bile bilmedim.

Garïp-xan, écoute mes soupirs !

Je ne pouvais pas savoir que tu reviendrais.

J'ai abandonné mon honneur et ma vergogne,

Je ne pouvais pas savoir que tu étais revenu.

D'autres versions, comme la version tatare de Crimée (publiée par Radloff en 1896) et la version azerbaïdjanaise, diffèrent d'une manière plus frappante, mais même là on peut observer des correspondances textuelles avec les versions turkmènes et karakalpakes. Le « refrain » du poème dans la version tatare de Crimée, par exemple, est très proche de celui de notre version : Dost, säniñ gäldigiñ bän duyamadïm, «Ami, je ne pouvais pas sentir que tu étais revenu $»^{30}$.

J'ai appelé G̈ärip et Sänem une épopée lyrique. En principe l'« épopée orale ", héroïque ou lyrique, est appelée dastan en karakalpak, du persan dāstān, " histoire ", " récit ", ainsi qu'en ouzbek (dāstān), en turkmène (dessan), en azerbaïdjanais (dastan), en ouighour (dästan), etc. ${ }^{31}$. Cependant la terminologie indigène des genres narratifs n'est pas très précise ${ }^{32}$. Aussi garderai-je ici cette imprécision et parlerai-je d'épopée orale ou de dastan sans me préoccuper du problème des genres ${ }^{33}$. Ce faisant, je ne cherche pas à minimiser les différences radicales qui existent entre un dastan comme Qoblan et un dastan comme Gärip-ašiq. Si, pour un lecteur français, Qoblan fait penser à une chanson de geste comme La Chanson de Roland, le dastan G̈ärip et Sänem évoque la chantefable de Aucassin et Nicolette. La ressemblance ne se limite d'ailleurs pas au contenu et au ton lyrique du dastan, mais touche à la forme même de l'épopée lyrique (souvent aussi de l'épopée héroïque). Ces dastan sont de véritables chantefables, c'est-à-dire que le récit en prose y alterne avec des parties en vers.

Cette alternance entre prose et poésie est, comme dans la chantefable, une alternance entre dire et chanter. La forme "prosimétrique» caractérise surtout les traditions turque de Turquie, azerbaïdjanaise, turkmène, ouzbèke, ouïghoure et karakalpake. En Karakalpakie cependant, existent aussi des œuvres entièrement en vers (venant de žiraw), mais ces dastan sont en minorité ${ }^{34}$. Dans les traditions kazakhe et kirghize, 
l'épopée en vers (octosyllabes) prédomine, bien que le dastan "prosimétrique » s'y rencontre aussi. Quant aux traditions turques de l'Altaï, les épopées y sont également composées en vers, mais ceux-ci se signalent par une plus grande irrégularité métrique que dans les traditions centrales ${ }^{35}$. En outre, la prose a souvent une forme rythmique. Dans l'épopée ouzbèke notamment, les passages en prose rimée (sadž) abondent ${ }^{36}$. Les dastan du cycle de Gorog̀li par exemple commencent en général par un passage en sadž :

Burungi otgan zamāninda, eli ozbak āmāninda, qibladan tumaninda, Taka-Yāwmitniñ elinda, Čambilbelniñ belinda, dawrānni surib, qirq yigitni yig̈ib, āltin piyālaga maylar quyib, kunda ertan čulāninga qoy-soqimlar soyib, yurtni yig̈dirib, āčni toydirib, qirq yigitga silāwsin ton kiydirib, Goroǵlibek otdi. ${ }^{37}$

Au temps jadis, quand le peuple ouzbek vivait dans la prospérité, vers le sud-ouest, chez les tribus des Taka et des Yāwmit, sur la crête du Čambilbel, vivait Goroğlibek, régnant en splendeur, rassemblant autour de lui quarante yigit [jeunes hommes], versant du vin dans des gobelets en or, abattant du matin au soir brebis et bœufs pour des banquets gracieux, ordonnant d'assembler le peuple, de rassasier les affamés et de vêtir les quarante yigit de manteaux en peau de lynx.

Ce passage révèle un autre trait typique de la forme linguistique de l'épopée turque, le parallélisme. On rencontre dans cette phrase treize unités syntaxiques, dont la dernière est la proposition principale (Goroglibek otdi, « Gorog̀libek vivait »). Le reste de la phrase consiste en cinq compléments circonstanciels (de temps et de lieu) marqués par le suffixe du locatif -da et en sept constructions "participiales ", marquées par le suffixe converbial -ip. L'homologie grammaticale est renforcée par des rimes et des assonances (zamāninda, āmāninda, tumaninda ; elinda, belinda ; yig̈dirib, toydirib, kiydirib). Il n'est pas difficile d'imaginer comment la prose rimée peut se transformer en vers réguliers. Le parallélisme grammatical se trouve aussi dans les passages en vers; il est particulièrement prononcé dans l'épopée des peuples turcs de l'Altaï et dans l'oloñxo iakoute. Viktor Žirmunskij (suivant les recherches sur la métrique turque de Tadeusz Kowalski) a fait l'hypothèse que la base de la versification turque est le parallélisme grammatical - facile dans des langues agglutinantes comme les langues turques -, hypothèse hautement vraisemblable, surtout quand on pense aux traditions sibériennes. Les plus anciens monuments en langue turque - les inscriptions runiques en ancien turc de la vallée de l'Orkhon (en Mongolie, datant du viII ${ }^{e}$ siècle) - montrent déjà une préférence pour les structures parallèles ${ }^{38}$.

D'un autre côté, les traditions turques ont aussi subi l'influence des «littératures islamiques » telles les littératures arabe et persane. Il est bien possible que la présence du sadž dans les dastan ouzbeks soit due à l'influence du conte « prosimétrique » arabe, bien connu grâce au recueil des Mille et Une Nuits. Ainsi, le premier document épique turc, Le Livre de mon grand-père Qorqut (Kitab-i Dedem Qorqut), est un cycle de contes "prosimétriques ». Les manuscrits de ce cycle datent du $\mathrm{xvI}^{\mathrm{e}}$ siècle et le texte a certainement subi l'influence islamique, mais la plupart des récits reprennent sans aucun doute des traditions épiques plus anciennes, remontant au temps où les Oghouzes se trouvaient encore en Asie centrale sur les rives du Syr-Daria (du Ix ${ }^{e}$ au XI siècle $)^{39}$.

Un de ces récits est le conte de Bamsï Beyrek. Il s'agit d'une version d'Alpamiiš, une des épopées turques les plus connues. La version ouzbèke du barde Fāzil Yoldāš-oğli (1872-1955) est célèbre à juste titre ; c'est dans ce dastan que l'épopée héroïque turque trouve, dit-on, son expression classique ouzbèke. Cependant, cette version n'est qu'une parmi la trentaine de versions ouzbèkes recensées ${ }^{40}$. En outre, on connaît aussi des 
versions kazakhes et karakalpakes, qui à quelques variations près ressemblent tant aux versions ouzbèkes qu'on peut regrouper tous ces textes (en ce que Žirmunskij nommait la « version Kongrat »). L'histoire d'Alpamǐš est également connue des peuples de l'Altaï (Alïp Manaš) ainsi que des Tatars et des Bachkirs, chez lesquels existent des contes populaires et des récits "prosimétriques " sur «Alpamša ». La structure narrative de cette épopée est bipartie. La première partie est une quête matrimoniale; dans la seconde, le héros est d'abord fait prisonnier, puis gagne la liberté et retourne dans sa patrie. D'après la version ouzbèke de Fāzil Yoldāš-oğli, le contenu du dastan est le suivant ${ }^{41}$ :

Parmi les Qongirāt vivent deux frères, Bāybori et Bāysari, qui règnent sur la tribu. Comme ils sont dépourvus d'enfants, les frères vont passer quarante nuits au tombeau du saint Šāh-i mardān. Le saint leur promet que leurs femmes auront des enfants, la femme de Bāybori un fils et une fille, la femme de Bāysari une fille. La prophétie du saint s'accomplit et la femme de Bāybori accouche d'un fils et d'une fille, la femme de Bāysari d'une fille. Le fils, appelé Hakimbek (et plus tard Alpāmiš, «parce qu'il est un alp, un héros »), étudie à l'école musulmane et explique un jour à son père la nécessité de donner l'aumône et de lever la zakāt, l'impôt prescrit par l'islam. Quand Bāybori exige la zakāt de son frère Bāysari, celui-ci se fâche et décide d'émigrer avec la moitié de la tribu au pays de Tāyči-khan, khan des Kalmouks.

Barčin, la fille de Bāysari, est courtisée dans sa nouvelle patrie par Qāradžān et ses frères, fils d'une vieille sorcière kalmouke, Surxāyil. Malgré son refus, la belle Barčin doit consentir à un mariage contre son gré, mais elle réussit à négocier un délai de six mois. Durant ce répit, Alpāmiš, appelé à son secours, se procure un cheval digne d'un héros et se met en route. Avant d'arriver au pays des Kalmouks, Alpāmiš rencontre Qāradžān, son rival, qui a été averti de l'approche du héros par un rêve. Au cours de la rencontre, Qāradžān est converti à l'islam et les deux jeunes hommes deviennent amis. Qāradžān fait même la demande en mariage pour Alpāmiš et participe, à la place d'Alpāmišs, à la course de chevaux imposée par Barčin. Quant aux autres épreuves exigées par la jeune fille - tir à l'arc, lancement du poids et lutte -, c'est Alpāmiš lui-même qui en sort victorieux. Après une bataille acharnée avec les soldats de Tāyči-khan, à laquelle les Kalmouks sont défaits, Alpāmiš et Barčin retournent dans la patrie du héros.

Bāysari préfère cependant rester dans le pays des Kalmouks. Décision fatale, car le khan kalmouk, afin de se venger de la victoire d'Alpāmiš, dépouille Bāysari de toutes ses richesses et en fait un gardien de chameaux. Quand Alpāmiš est averti de l'infortune de son beau-père, il se rend une nouvelle fois au pays des Kalmouks. Mais en route la vieille Surxāyil, qui a perdu presque tous ses fils dans le combat avec Alpāmiš et dont le seul fils encore en vie, Qāradžān, est devenu l'ami de son ennemi, tend un piège au héros et à ses compagnons. Ils sont d'abord enivrés puis tués, à l'exception d'Alpāmiš, qui est jeté dans le zindān (prison souterraine).

Un jour, une oie sauvage, blessée, tombe dans le cachot d'Alpāmiš. Une fois qu'elle est guérie, Alpāmiš la prie d'aller porter un message dans sa patrie. Qāradžān accourt dès qu'il reçoit cet appel au secours, mais il est trop faible pour délivrer le héros. Par l'intermédiaire de Kayqubād, Alpāmiš attire l'attention de Tāwka, la fille de Tāyči-khan. Elle lui promet son secours et amène Bāy-Čibār, le cheval d'Alpāmišs. Bāy-Čibār réussit à tirer le héros du cachot avec sa queue. Avertis de la délivrance d'Alpāmiš, Tāyči-khan et ses guerriers kalmouks accourent, mais ils sont défaits malgré leur supériorité en nombre. Alpāmiš institue Kayqubād khan des Kalmouks et l'unit en mariage à la princesse Tāwka.

Après ces sept ans passés dans le zindān, Alpāmiš retourne au pays des Qongirāt. Mais entretemps la situation a changé : Ultāntāz, un fils de Bāybori par une esclave, règne maintenant sur les Qongirāt. C'est un despote qui a précipité la famille d'Alpāmiš dans le malheur. Il exige de Barčin qu'elle l'épouse et c'est en pleins préparatifs des noces qu'Alpāmiš arrive dans sa patrie. Comme Gärip et Ulysse, Alpāmiš rentre donc à point nommé, juste avant le remariage de son épouse. Il 
échange ses vêtements avec son brave serviteur Qultāy, qui reconnaît son maître à la marque que la main du saint Šāh-i mardān avait laissée sur l'épaule droite d'Alpāmiš. Malgré son déguisement, Alpāmiš est reconnu par sa grand-mère, par ses parents et par sa sœur. Arrivé au festin des noces, Alpāmiš échange des chansons improvisées avec sa femme, qui révèle dans ses vers sa fidélité à son mari. Quand le grand arc d'Alpinbiy, grand-père du héros, est apporté, Alpāmiš est le seul invité à parvenir à le bander. Son identité est finalement découverte et l'usurpateur exécuté. Alpāmiš est encore une fois réuni avec sa femme, son fils Yādgār (né après le départ du héros), le reste de sa famille et ses amis, en particulier Qāradžān.

Au cœur de l'épopée héroïque se tient le héros qui montre sa prouesse et accomplit de hauts faits, grâce à son cheval et à ses compagnons ${ }^{42}$. Dans nombre de ces épopées relevant des traditions centrales, l'ennemi classique est le Kalmouk. Ce fait se fonde historiquement sur les invasions en Asie centrale des Ö̈rates (Mongols occidentaux), qui, à partir de la fin du $\mathrm{xvI}^{\mathrm{e}}$ siècle, migrent en un trajet sanglant depuis la Dzoungarie jusqu'aux steppes d'entre la Volga et le fleuve Oural ${ }^{43}$. Cela ne veut pas dire que les sujets épiques soient tous postérieurs au XvI ${ }^{\mathrm{e}}$ siècle, bien au contraire, comme Alpamiǐ le démontre. C'est l'épopée d'une société nomade. Le point de référence de l'action est le clan et la tribu. Le héros ne lutte pas seulement pour lui-même, mais aussi pour la survie de son clan et de sa tribu. Il y a là une différence fondamentale entre l'épopée héroïque et le dastan lyrique et aventureux. Ce qui incite à lier morale héroïque et vie nomade, c'est que l'épopée héroïque est un genre sinon mort, du moins moribond, dans les sociétés turques sédentaires, alors qu'elle s'est conservée jusqu'à nos jours chez les Karakalpaks, les Kazakhs et les Kirghizes, peuples d'origine nomade par excellence ${ }^{44}$.

Parmi les plus célèbres épopées turques en Asie centrale, il faut aussi mentionner deux cycles importants, celui de Gorogili et celui de Manas. Le premier a connu une diffusion si vaste en Asie centrale et au Proche-Orient qu'il s'est propagé même parmi les peuples voisins non turcs. On trouve donc des épopées et des récits sur Gorogili et ses compagnons en arménien, géorgien, kurde, arabe (de Boukhara) et tadjik aussi bien qu'en turc (de Turquie), azerbaïdjanais, turkmène, ouzbek, ouïghour, tatar, karakalpak et kazakh ${ }^{45}$. Dans les versions azerbaïdjanaises et turques (de Turquie), Gorogili - qui s'appelle ici Köroğlu - est un proscrit et un bandit. Il est le fils du maître des chevaux du sultan, qui fut injustement aveuglé : de là son nom "Köroğlu », "fils d'aveugle ». Avec sa troupe de quarante compagnons, il accomplit de nombreux faits d'armes, racontés dans les branches diverses du cycle (appelées kol, «bras », en turc). Dans les versions qui ont cours en Asie centrale, «le fils de l'aveugle » (kör) devient «le fils du tombeau » (gör, gor) : le héros Goroggli (Görogli), né dans le tombeau de sa mère, est sauvé miraculeusement et devient un puissant khan. Que l'action soit transférée de Goroġli à ses fils adoptés Awaz et Hasan et à leurs enfants et même petits-enfants est typique des versions d'Asie centrale, surtout des versions ouzbèkes. Certaines branches sont elles-mêmes des épopées à rallonges. Le dastan ouzbek de Rawšan, par exemple, où figure un petit-fils de Gorogili, comporte, dans la version du baxši Ergaš Džumanbulbuloğli, 62 passages en vers, soit un total de 5140 vers. Le manuscrit, qui fut écrit entre 1927 et 1928, compte plus de 400 pages en écriture arabe ${ }^{46}$.

29 Le cycle de Manas, en revanche, n'est pas répandu ailleurs que chez les Kirghizes ${ }^{47}$. Manas est, comme les épopées homériques, une épopée au sens hégélien : elle exprime d'une manière poétique les idéaux et les aspirations, les mœurs et les traditions, les habitudes et la conception du monde d'un peuple entier. Manas est l'épopée des superlatifs : non seulement « épique » au sens hégélien, mais aussi au sens courant du 
mot, c'est-à-dire que c'est un récit interminable, qui abonde en actes de bravoure et en faits héroïques. Au Kirghizistan même, un corpus énorme a été collecté : on parle de plus de deux millions de vers. Les deux bardes les plus célèbres sont Sagïmbay Orozbaqov (1867-1930) et Sayaqbay Qaralaev (1894-1971). La version de Manas de Sagïmbay comporte plus de 180000 vers; celle de Sayaqbay en a 500553 et traite de Manas, de son fils Semetey, de son petit-fils Seytek et des fils de ce dernier. Le cycle de Manas se rencontre aussi chez les Kirghizes qui vivent hors du Kirghizistan. Au Xinjiang, la version la plus étendue, transcrite de la bouche du barde Džüsüp Mamay (né en 1918), comporte environ 210000 vers et huit branches, Manas, Semetey, Seytek, Keninim, Seyt, Asilbača-Bekbača, Sombilek et Čigitey ${ }^{48}$.

Comme d'autres héros de l'épopée et du conte turcs, Manas est le fils d'un couple resté sans enfant jusqu'à un âge avancé. Quand un fils naît enfin à Džaqïp-khan et à sa femme Čìïrči dans leur vieillesse, quatre prophètes apparaissent et lui donnent le nom de «Manas»:

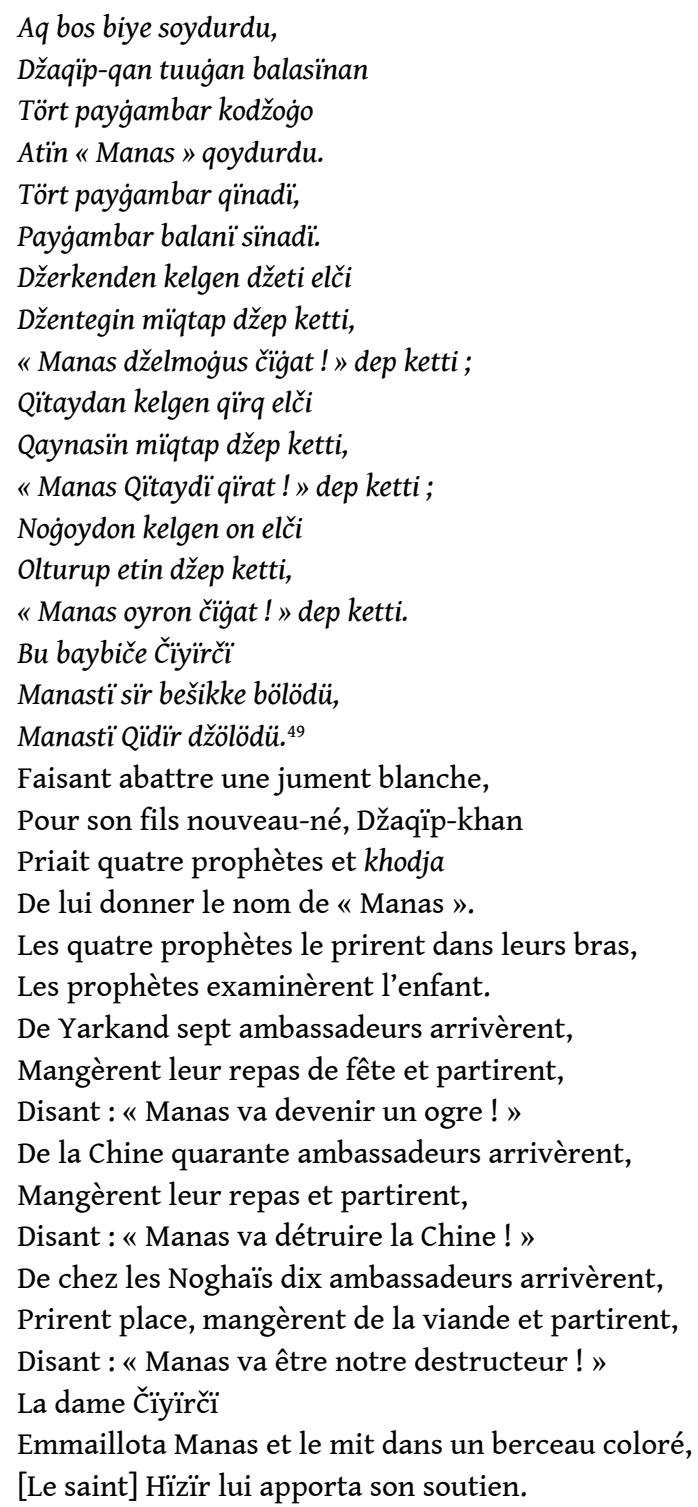

31 Manas accomplit les prophéties prononcées à sa naissance; il réussit à vaincre ses ennemis au sein de sa tribu et en dehors. Comme d'autres héros de l'épopée héroïque, 
Manas possède une stature impressionnante et une force surnaturelle; encore au berceau il proclame son intention de lutter contre les infidèles :

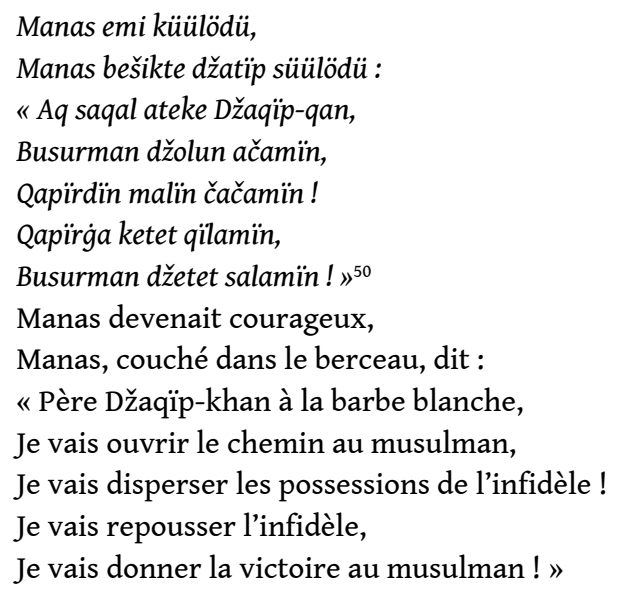

Pendant ses expéditions, Manas est accompagné de ses quarante compagnons, mais il partage aussi beaucoup d'aventures avec son ami kalmouk Almambet, le fils de Qarakhan des Ö̈rates. Almambet avait été converti à l'islam et jouissait de l'hospitalité du khan kazakh Kökčö. Les compagnons de Kökčö, qui enviaient la position de favori d'Almambet, le diffamèrent. Quand Kökčö accorda créance à ce bruit, Almambet partit trouver Manas. Celui-ci, prévenu de l'arrivée d'Almambet par un songe, alla à sa rencontre et les deux héros devinrent amis. Ils scellèrent leur amitié en buvant le lait qui jaillit miraculeusement des seins de Čïyïrčì. À cause de l'inimitié de Kökčö, Manas fut d'abord amené à livrer un duel acharné à Kökčö, puis fut trahi et empoisonné. Tandis que les quarante compagnons de Manas ne faisaient guère preuve de loyauté à son égard, son faucon, son cheval et son chien restaient fidèles à leur maître et, par leur conduite, ils poussèrent ainsi Dieu à ramener le héros à la vie. Ce ne fut pas la seule fois que Manas revint de la mort. Lorsqu'il partit plus tard lutter contre les Kalmouks, il ne se soucia pas de l'avertissement de sa femme Qanikey et fut à nouveau empoisonné, mais cette fois ce furent les facultés magiques de celle-ci qui le ramenèrent à la vie. Cependant, même un héros puissant comme Manas doit mourir ; et, en effet, il trouve la mort par la main de Qoñurbay, son ennemi juré chinois. Heureusement il lui reste un héritier digne de lui, Semetey, son fils vaillant.

Voilà, résumée en quelques traits, la première partie du cycle épique. Des « épisodes » comme la quête de la belle Qanïkey, fille de Temir-khan, ou la grande expédition de Manas à Pékin pour lutter contre les Chinois commandés par Qoñurbay sont des épopées en soi. Ainsi, la "branche de Qanïkey " de la version de Sagïmbay comporte environ 4000 vers. Autre exemple, la première branche du cycle de Manas à avoir été mise par écrit est un poème épique d'environ 3000 vers, La cérémonie commémorative pour Kökötöy-khan. Cette épopée, mise par écrit en 1856 par le prince kazakh Chokan Valikhanov, dépeint la cérémonie que Boqmurun, fils de Kökötöy, un vieux compagnon de Manas, donna en l'honneur de son père mort. La nature archaïque de cette épopée est soulignée par les nombreuses ressemblances qu'on relève entre la compétition organisée à l'occasion de la cérémonie commémorative pour Kökötöy et les jeux funèbres qui eurent lieu après la mort de Patrocle dans L'Iliade.

J'ai qualifié Manas de cycle épique parce que les épisodes de la vie de Manas constituent des épopées complètes, mais aussi parce que l'épopée s'étend au delà de la vie du héros à celle de ses enfants, de ses petits-enfants et arrière-petits-enfants - et même dans 
quelques versions jusqu'à la quatrième génération. Toutes les branches du cycle ne sont pas de nature héroïque. Dans Semetey, une grande partie de l'action est consacrée aux aventures amoureuses du héros épris de la fée Ayčürök, ce qui donne un caractère plus lyrique à l'épopée. Dans les branches ultérieures du cycle, notées au Xinjiang, des éléments fabuleux et des motifs rappelant les contes de fées occupent le premier plan. Mais cette variété même est la marque d'une grande épopée : par sa texture variée, elle reflète poétiquement la richesse et la complexité de la culture matérielle et spirituelle d'un peuple.

Comme le cycle de Manas, l'œuvre épique des peuples turcophones est un tout volumineux à caractère multiforme. Et nous ne pouvons aborder ici que quelques problèmes choisis. La question qui est au centre des chapitres suivants est celle du sens : les épopées sont-elles douées d'une signification liée à l'idéologie ou peut-être à l'inspiration religieuse et au culte, ou ont-elles plutôt une signification due à leur fonction sociologique ou à la structure poétique du texte récité, chanté, mis en scène? Ces questions ne se posent pas uniquement pour l'épopée orale turque mais concernent toute épopée orale, et il faudrait donc faire référence ici aux théories sur l'épopée orale et l'oralité ainsi qu'aux œuvres comparatives qui traitent de poésie épique. Cependant, même si l'orientation des chapitres suivants est comparative, cette référence ne pourra être, dans le cadre de la présente étude, que très sommaire ${ }^{51}$.

Pour parler de l'épopée orale des peuples turcs, j'emploie «le présent ethnologique ». Mais il faut admettre que, de nos jours, l'épopée orale n'est plus qu'une curiosité muséologique ou folklorique dans bien des régions turcophones. Dans d'autres régions, elle est moribonde, et c'est seulement dans les lieux les moins accessibles que la tradition orale a pu se conserver jusqu'à nos jours. Le barde karakalpak dont j'ai parlé plus haut, Žumabay-žiraw, est le dernier žïraw karakalpak à avoir appris son répertoire par la voie orale traditionnelle. Après lui, il n'y aura plus que des artistes folkloriques. Cette situation se retrouve à peu près partout. Les changements politiques des dernières années dans l'ex-URSS ont encore accéléré ce processus et l'on voit venir la fin de la tradition orale de l'épopée, fin déjà prédite par Hermann Vámbéry au milieu du XIX siècle :

Terakki, c'est-à-dire le progrès, est devenu le mot de ralliement de tout un chacun, malgré les entreprises strictement conservatrices des mollahs fanatiques.

Dans ces circonstances, il est hors de doute que l'ancien mode de vie des peuples d'Asie centrale avec ses formes sauvages et pittoresques et sa vision particulière du monde aura bientôt entièrement disparu. ${ }^{52}$

On doit se réjouir que Vámbéry se soit trompé sur la date : on peut encore entendre la voix du barde traditionnel. Mais il est douteux que la tradition vivante se perpétue encore longtemps. Heureusement, un grand nombre de textes ont été recueillis et mis par écrit au cours du $\mathrm{xx}^{\mathrm{e}}$ siècle ; cependant, un bien plus grand nombre de textes versions d'épopées connues ou inconnues - sont hélas à jamais perdus ! 


\section{NOTES}

1. Cf. Martinet 1987. Pour la transcription des langues turques, j'ai suivi le système utilisé dans le premier volume des Philologiae Turcicae Fundamenta (Deny et al. 1959, p. xiv sq.), avec les exceptions suivantes : au lieu de $\langle\gamma>$ j'emploie $<\dot{\mathrm{g}}>$; au lieu de $<x>$ j'emploie $<x>$; au lieu de $<\breve{\mathrm{g}}>$ pour l'affriquée palato-alvéolaire sonore j'emploie $\langle\mathrm{dž}>$; au lieu de $\langle\mathrm{y}\rangle$ pour la nasale vélaire, j'emploie $<\tilde{n}>$, signe utilisé dans la transcription latine des langues turques en Turquie et en Asie centrale. Le turc de Turquie est écrit dans son orthographe latine habituelle. Les mots arabes sont transcrits conformément au système de transcription utilisé par les arabisants (cf. par ex. Lecomte et Ghedira 1960-1961), avec les exceptions suivantes : comme pour les langues turques, la fricative vélaire sourde est transcrite $<\mathrm{x}>$ et l'affriquée palato-alvéolaire sonore $<\mathrm{dž}>$. Dans les textes en langues turques, j'ai transcrit les mots arabes d'après la prononciation turque. Le «a» ouvert de l'ouzbek et du persan (phonétiquement [э:]) est transcrit $<\overline{\mathbf{a}}>$. Les mots russes sont transcrits conformément au système employé par les linguistes. Quant aux termes géographiques, ethnologiques, historiques et linguistiques, j'ai adopté l'orthographe qui se trouve dans l'édition de 1995 du Dictionnaire Hachette encyclopédique illustré ; pour les noms et les termes relatifs à l'Asie centrale, j'emploie aussi les formes qui se trouvent dans Fourniau 1994. Toutes les traductions d'une langue turque, du russe ou de l'allemand sont miennes, hormis les cas où les traducteurs sont expressément nommés.

2. Cf. le sous-titre de l'Histoire des Turcs de Jean-Paul Roux, Deux mille ans du Pacifique à la Méditerranée (1984a).

3. Il va sans dire que ce chapitre d'introduction ne peut aborder toutes les questions qui traitent de l'origine des langues turques (lieu et date), de leur parenté présumée avec les langues mongoles et toungouses et de leur classification génétique. Sur l'origine, le regroupement et le développement des langues turques, cf. Deny et al. 1959; Menges 1995. Sur l'ethnogenèse et l'histoire des peuples turcs (turcophones), cf. Roux 1984a; Golden 1992. Sur la civilisation et la religion des anciens Turcs, cf. aussi Klimkeit 1988.

4. Pour un traitement d'ensemble de l'épopée orale turque, cf. Boratav 1964; Chadwick et Zhirmunsky 1969 ; Žirmunskij 1974a ; Bašgöz 1978 ; Reichl 1992. Sur l'âge héröque des Kazakhs et des Karakalpaks, cf. chap. IV.

5. Cette version fut enregistrée en septembre 1994 à Alma-Ata lors d'un congrès de musicologie consacré à la musique des peuples turcs. J'ai enregistré deux versions complètes de l'épopée du même barde, l'une en 1990, l'autre en 1993 (à Noukous en Karakalpakie). La version de son maître Esemurat-žiraw est éditée dans Žapaqov et al. 1981; le passage correspondant se trouve pp. 148-149.

6. Je reviendrai sur Qoblan au chapitre IV, où l'on trouvera une analyse plus détaillée de cette épopée.

7. Pour l'arbre généalogique de l'«école de Kattaqorgān» du XVII ${ }^{\mathrm{e}}$ au milieu du Xx $\mathrm{x}^{\mathrm{e}}$ siècle, tracé par Hādi Zarif, cf. Zarif 1970, p. 41. La tradition ouzbèke est traitée à fond dans le livre de Žirmunskij et Zarifov 1947 ; sur les Ouzbeks du nord de l'Afghanistan, cf. Dor 1977.

8. Des renseignements sur ces bardes se trouvent (en karakalpak) dans Ayïmbetov 1988, p. 79 sq.; Maqsetov 1983, p. 8 sq. (Soppaslï Sïpïra-žïraw), p. 26 sq. (Nurabulla-žïraw), p. 77 sq. (Esemuratžiraw).

9. En kazakh, le mot žïraw se rencontre à côté du terme aqün; bien que les deux termes soient à peu près synonymes et fassent référence au barde traditionnel, le mot žiraw est plus archaïque ; cf. Ismailov 1957, pp. 43-48. Le mot žïrši (aussi dérivé de žir), d'autre part, désigne en kazakh un barde qui a mémorisé des poèmes au lieu de les acquérir au cours d'un apprentissage ; cf. ibid. pp. 48-56. En kirghize, comme en kazakh, le terme général dénotant le barde (et aussi le poète) 
est aqün; on trouve également le terme ïrčï, qui correspond au kazakh žirši ; cf. Judaxin 1985, I, p. 42 (s.v. aqïn) ; II, p. 439 (s.v. ïrči) ; Dor 1982, p. 11 ; pour les autres termes employés en kirghize pour les bardes, voir ci-dessous.

10. La dérivation du mot turc (et mongol) du chinois est proposée par A. von Gabain 1974, p. 326. 11. V. V. Illarionov, dans son étude sur l'art des bardes iakoutes, écrit qu'au nom de bardes célèbres les Iakoutes joignaient les mots oloñhosut ou ïrïa (ce dernier étant, comme ïrči ou žiraw, une dérivation de ïr) en qualité de titre honorifique ; Illarionov 1982, p. 13 sq.

12. Une désignation plus ancienne est džomoqču, dérivation de džomoq, mot qui signifie aussi bien le conte populaire que l'épopée hérö̈que. Pour le chanteur de Semetey, cycle de poèmes épiques consacrés aux aventures de Semetey, fils de Manas, on emploie le terme semeteyči. Il existe aussi d'autres termes pour le barde chez les Kirghizes, ainsi que différents types de manasči, tels le čoñ manasči «le grand (ou vieux) manasči», le ürönčük manasči «le manasči élève», etc.; cf. l'article «Manasčï» dans l'encyclopédie Manas (Qarïpqulov et al. 1995, II, pp. 68-76) ; Gölgeci 1995 ; cf. aussi Žirmunskij 1961, pp. 87-88; Musaev 1979, p. 31 sq. Chez les Kirghizes du Pamir afghan (transplantés en Anatolie orientale) se rencontre aussi le terme afez, apïz (de l'arabe ḥăfiz, titre honorifique conféré à qui a mémorisé le Coran) ; cf. Dor 1976.

13. Sur le qobïz, cf. Karomatov 1972, p. 107 sq. ; Slobin 1976, p. 248 sq.

14. Sur la dombira ouzbèke, cf. Karomatov 1972, p. 116 sq. ; Slobin 1976, p. 212 sq.

15. Sur le dutar, cf. Sachs 1913, p. 124 (s.v. Dutâr) ; Karomatov 1972, p. 123 sq. ; Slobin 1976, p. 224

sq.

16. Sur la dombra kazakhe, cf. Beliaev 1975, p. 83 sq. ; Elemanova et al. 1993, p. 67 sq.

17. Les cordes du topšuur sont généralement pincées, mais on peut aussi jouer de l'instrument avec un archet. Cf. Šul'gin 1968, p. 53 sq. ; 1973, p. 455 sq.

18. Sur le gidždžak, cf. Karomatov 1972, p. 110 sq. ; Slobin 1976, p. 243 sq. ; sur la récitation du baxši khorezmien, cf. Abdullaev 1989, p. 115 sq. ; Reichl 1985a, p. 625 sq.

19. Pour une description de la manière de réciter/chanter l'épopée, cf. chap. $v$.

20. Sur la métrique de la poésie populaire turque, cf. Boratav 1964, p. 11 sq. ; Xamraev 1969, p. 64 sq.

21. Pour les versions karakalpakes, cf. Maksetov et al. 1977, pp.59-68. Une des ces versions, transcrite de la bouche du baqsï Eššan Qospolatov dans les années 1930 et 1934, est éditée dans Alïmov et al. 1985, pp. 15-187. Le passage correspondant à l'extrait cité ci-dessus se trouve dans le même ouvrage, pp. 169-170.

22. Cf. Boratav 1964, p. 30 sq. Une version turque a été éditée par Güney 1958 ; une version azerbaïdjanaise se trouve dans Tähmasib et al. 1979, pp. 167-202. Deux versions tatares de Crimée sont publiées dans Radloff 1896, pp. 241-297, et Bekirov 1980, pp.101-109. On peut lire une version ouïghoure dans Raxman 1981, pp. 237-297, et une version turkmène dans Aširov et Xayïdov 1958, pp. 123-143. Pour les versions ouzbèkes, cf. Žirmunskij et Zarifov 1947, p. 294. La traduction russe de Lermontov se trouve dans l'édition de Moscou de 1975-1976, IV, pp. 324-331.

23. Cf. le motif $\mathrm{n}^{\circ} \mathrm{K} 1815.1$ Return home in humble disguise et les motifs suivants dans Thompson 1955-1958. Le nom du héros de cette épopée prend des formes différentes selon la langue turque considérée ; en kazakh et en karakalpak la forme du nom est «Alpamïs», en ouzbek «Alpāmišs», etc. ; j'emploie la forme «Alpamǐš», la plus courante dans la littérature critique.

24. Sur ce baqsï, cf. Maqsetov 1983, pp. 188-196.

25. Une transcription de cette mélodie, avec deux strophes du poème, se trouve dans Šafrannikov et Beljaev 1959, pp. 153-154.

26. Cf. Vámbéry 1867, p. 95 sq. ; une édition complète par Vámbéry parut en 1911. Sur ce récit, cf. chap. II. Le mot qissa-xān est dérivé de l'arabe qișṣa «conte, récit» et du persan xāndan «lire». Sur le qissa-xān ouzbek, cf. Žirmunskij et Zarifov 1947, pp. 28-29: «Dans les villes et les villages près des villes, il y avait aussi des récitants professionnels (appelés qissa-xān), qui lisaient à haute voix des livres populaires à l'intention d'une population analphabète. Ils récitaient dans les bazars ou, 
quand on les invitait, dans des maisons privées. À ces occasions, un lecteur expérimenté récitait le texte par cœur, avec des variantes individuelles. Le répertoire musico-poétique des chanteurs populaires (ašulači) comportait des œuvres de poésie et de musique ouzbèkes classiques. C'est par cette voie qu'en ouzbek la littérature écrite a influencé depuis longtemps la poésie épique populaire.»

27. Cf. Ayïmbetov 1988, p. 130 sq.

28. Les formes žar, menen, etc., relèvent de la langue littéraire ; la situation dialectale est plus compliquée ; il y a des dialectes karakalpaks «turkménisés» et d'autres «ouzbékisés»; cf. Nasyrov 1983.

29. Ašïrov et Xayïdov 1958, p. 142. Une version ouzbèke de ce récit, qui ressemble beaucoup à ce texte turkmène, a été enregistrée (Melodija, disque no D-014545-50), narrée et chantée par le baxši Rozimbek Murādov; ici le barde (qui d'ailleurs n'appartient plus à la tradition orale) joue du tar (un instrument à cordes pincées, en forme de poire) et est accompagné par un ensemble de quatre musiciens.

30. Radloff 1896, pp. 293-294.

31. Cf. Elçin 1967.

32. Cela ne veut pas dire, naturellement, que les spécialistes du folklore de l'Asie centrale n'aient pas développé une terminologie et une classification plus précises. Ainsi, la classification ouzbèke distingue entre «épopée héroïque» (qahramānlik dāstān), «récit de guerre» (džañnāma-dāstān), «épopée historique» (tarixiy dāstān), «épopée lyrique» (romanik dāstān) et «épopée de provenance littéraire» (kitābiy dāstān) ; cf. Mirzaev et Sarimsāqov 1981.

33. J'ai discuté le problème des genres épiques ailleurs ; cf. Reichl 1992, pp. 119-141 sq.

34. Ainsi il existe dans les archives de l'Académie des sciences à Noukous une version karakalpake de l'épopée Qoblan qui est entièrement en vers. Sur la forme «prosimétrique» dans la littérature mondiale, cf. Harris et Reichl 1997.

35. La métrique de l'épopée altaïenne est décrite par Radloff 1866a, p. 109 sq.

36. Cf. Sarimsāqov 1978.

37. Zarif 1981, p. 5 (Xāldārxān, par le baxši Ergaš Džumanbulbul-og̉li).

38. Cf. Žirmunskij 1985 ; sur le parallélisme dans la poésie populaire turque, cf. aussi Reichl 1994 (avec bibliographie).

39. Sur le Livre de mon grand-père Qorqut, cf. Rossi 1952 ; Lewis 1974. La datation des épopées orales est extrêmement difficile et controversée. Dans le cas d'Alpamǐ̌s, la présence d'une version turque (oghouze) atteste l'existence de l'épopée dès la fin du Moyen Âge. Quant à dire que les Oghouzes ont importé l'épopée d'Asie centrale en Anatolie... ce point est contesté ; à mon avis, les Oghouzes ont eu connaissance de la légende d'Alpamǐs quand ils étaient encore en Asie centrale, hypothèse plus probable que celle consistant à postuler une influence plus tardive d'est en ouest (ou d'ouest en est).

40. Cf. Mirzaev 1968 ; Žirmunskij 1960.

41. D’après l'édition de Zarif, Mirzaev et Hakimdžānova 1992-1993.

42. Sur les motifs caractéristiques de l'épopée héroïque des peuples turcs (et mongols), cf. Lipec 1984.

43. Cf. Grousset 1965, pp. 599-602.

44. Cf. chap. III.

45. Cf. l'étude de Karryev (1968), dans laquelle les versions les plus importantes sont énumérées et décrites.

46. J'ai publié une traduction allemande de Rawšan (cf. Reichl 1985b). Pour une traduction française de divers «dāstāns» ouzbeks sur Nurali, autre petit-fils de Gorogili, cf. Dor 1991; sur le cycle de Gorogili, cf. ibid. p. 161 sq.

47. J'ai entendu parler en Chine de contes kazakhs en prose sur Manas qui circuleraient chez des Kazakhs du Xinjiang. 
48. Sur Manas, cf. l'étude de Bogdanova, Žirmunskij et Petrosjan 1961. Sur toutes les questions relatives à l'épopée, voir l'encyclopédie détaillée sur Manas (Qarïpqulov et al. 1995). Je ne citerai que quelques exemples d'éditions (et de traductions) : Hatto 1977 pour une édition critique du texte de Valikhanov (avec une traduction anglaise); l'édition de plusieurs parties du cycle (accompagnée d'une traduction allemande) par Radloff 1885, rééditée avec une traduction anglaise et des commentaires de Hatto 1990 ; la version du barde Sagïmbay Orozbaqov éditée (avec une traduction russe et des commentaires) dans la série «Êpos narodov SSSR» en quatre volumes ; cf. Sadykov et al. 1984-1990.

49. Hatto 1990 , p. 6.

50. Hatto 1990 , p. 7.

51. Voir surtout les études comparées de H. M. et N. N. Chadwick 1932-1940, de Bowra 1952, et de Hatto et Hainsworth 1980-1989.

52. Vámbéry 1911, p. 4.

\section{RÉSUMÉS}

Le premier chapitre offre une courte introduction à l'épopée orale turque d'Asie centrale. Parmi les diverses traditions de poésie orale des peuples turcs, c'est aux épopées de ce que l'on nomme les «traditions centrales» que ce chapitre et les suivants sont consacrés, c'est-à-dire à la poésie orale des Ouzbeks, des Ouïghours, des Kazakhs, des Karakalpaks et des Kirghizes. On distingue dans ces traditions différents types de bardes : baxši, aqïn, žiraw, manasči, etc. ; ce sont en général des professionnels qui ont acquis leur art et leur répertoire au cours d'un apprentissage plus ou moins formel auprès d'un ou de plusieurs maîtres bardes. Bien que l'on relève maintes ressemblances entre ces traditions, elles connaissent néanmoins une assez grande diversité quant à la forme, au genre et au mode d'exécution des épopées. Les épopées peuvent être en vers, elles peuvent être en un mélange de vers et de prose; les vers peuvent être de huit syllabes, souvent allitérés, ou de onze ou douze syllabes, souvent rimés ; le répertoire du barde comporte à la fois des épopées héroïques et des épopées lyriques (dastan) ; le barde peut exécuter l'épopée en chantant sans l'accompagnement d'un instrument (tel le manasči kirghize), il peut s'accompagner lui-même sur un instrument à cordes pincées ou jouées à l'archet, et il peut aussi être accompagné par un autre musicien, voire par un petit ensemble.

The first chapter provides a short introduction to the Turkic oral epic of Central Asia (Bref aperçu de l'épopée orale turque d'Asie centrale). Among the various traditions of Turkic oral poetry, this and the following chapters focus on the epics of what is termed the «central traditions», i.e. the oral poetry of the Uzbeks, Uighurs, Kazakhs, Karakalpaks and Kirghiz. In these traditions different types of singers can be distinguished: baxši, aqïn, žiraw, manasči and others ; these singers are in general professionals who have acquired their art and their repertoire in the course of a more or less formal training with one or more master singers. Although there are many similarities between these traditions, there is also a fair amount of variety as to the form, the genre and the manner of performance of the epics. Epics can be in verse, they can be in a mixture of verse and prose; the verse can be in octosyllabic lines, often alliterating, or in lines of eleven/twelve syllables, often rhyming; in the singers' repertoire there are both heroic epics and lyrical love romances (dastans) ; the singer might perform the epic in chanting without the accompaniment 
of an instrument (as the Kirghiz manasči), he might accompany himself on a plucked or bowed instrument, and he might be further accompanied by another musician or even a small ensemble.

\section{INDEX}

nomsmotscles Karakalpak, Kazakh, Kirghiz, Ouzbek, Turc

Thèmes : islam, littérature, mythologie, oralité

Mots-clés : Asie centrale, barde, épopée, métrique, poésie

\section{AUTEUR}

\section{KARL REICHL}

Karl Reichl est professeur de philologie anglaise à l'Université de Bonne (Allemagne). Il a publié de nombreux travaux sur la littérature orale des peuples turcs : Turkic Oral Epic Poetry: Traditions, Forms, Poetic Structure (1992), The Oral Epic: Performance and Music (2000), Singing the Past. Turkic \& Medieval Heroic Poetry (2000), Edige: A Karakalpak Oral Epic (2007). 